\title{
Research Paper: Comparison of the Position Sense of the Knee Joint in Patients With Multiple Sclerosis and Healthy Controls
}

\author{
Saina Aliabadi1 ${ }^{1}$, Roya Khanmohammadi ${ }^{1}$, Gholamrezareza Olyaei ${ }^{1}$ (D), Nastaran Ghotbi ${ }^{1}$ (D), Saeed Talebian ${ }^{1}$ (D, Abdolreza Naser Moghadasi ${ }^{* *}$ (D)
}

1. Department of Physiotherapy, School of Rehabilitation, Tehran University of Medical Sciences, Tehran, Iran.

2. Multiple Sclerosis Research Center, Sina Hospital, Tehran University of Medical Sciences, Tehran, Iran.

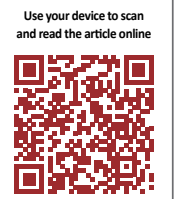

Cftration: Aliabadi S, Khanmohammadi R, Olyaei Gh, Ghotbi N, Talebian S, Moghadasi AN. Comparison of the Position Sense of the Knee Joint in Patients With Multiple Sclerosis and Healthy. Journal of Modern Rehabilitation. 2019; 13(1):59-64. http://dx.doi.org/10.32598/JMR.13.1.59

http://dx.doi.org/10.32598/JMR.13.1.59

Article info:

Received: 10 Aug 2018

Accepted: 27 Nov 2018

Available Online: 01 Jan 2019

\section{Keywords:}

Multiple Sclerosis (MS),

Position sense, Proprioception, Knee joint, Repositioning error

\begin{abstract}
Introduction: Position sense, one of the most accurate senses in the body, makes everyone aware of the state of the body in space. This sense is an essential ability in maintaining physical health and avoiding injury. Deficits in position sense cause balance impairments in people with mild Multiple Sclerosis (MS). Position sense requires instant and coordinated communication between the central nervous system and peripheral nervous system, while in patients with MS, communication between the brain and other parts of the body is disrupted. This study aims to compare the position sense of knee joint in people with MS and healthy subjects.
\end{abstract}

Materials and Methods: Ten healthy subjects with the Mean \pm SD age of $27.6 \pm 3.71$ years and 10 persons with MS disease and the Mean \pm SD age of $31.40 \pm 3.50$ years participated in this study. For evaluating their position sense of knee joint, they flexed their knees (from 90 to 45 degrees) four times, and then a software calculated their repositioning errors.

Results: No significant changes in repositioning errors (constant, variable, absolute) were observed in MS patients, and the control group $(\mathrm{P}>0.05)$

Conclusion: The results indicate that mild MS disease cannot disturb the position sense of knee joint.

\section{Introduction}

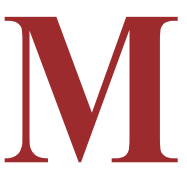

ultiple Sclerosis (MS) is a Central Nervous System (CNS) autoimmune disease that attacks myelin sheaths of nerve fibers and disrupts communications between the brain and other parts of the body [1]. About 2.5 million adult people have MS in the world. Iran is ranked first in the Middle East in the number of MS patients. According to reports of Iran Multiple Sclerosis Society, 70000 people have MS in Iran. Currently, Isfahan Province holds the record of the prevalence of MS in the country [2].

The national MS society has categorized it into four main groups: Relapsing-Remitting MS (RRMS), Pri-

* Corresponding Author:

Abdolreza Naser Moghadasi, MD.

Address: Multiple Sclerosis Research Center, Sina Hospital, Tehran University of Medical Sciences, Tehran, Iran

Tel: +98(912)8346907

E-mail:s_aliabadi@razi.tums.ac.ir 
mary-Progressive MS (PPMS), Secondary Progressive MS (SPMS), and Progressive-Relapsing MS (PRMS). The most common type of MS is RRMS. According to the national institute of health, $90 \%$ of MS patients have relapsing-remitting type [2].

Although MS usually is not a fatal illness [2], patients with MS experience symptoms such as functional impairments, fatigue, muscle weakness, decreased cognition, and spasticity. Generally, falls and gait impairments are related to lower position sense, which is the most critical complaints in patients with MS in their daily life [3]. Disruption of joint position sense could lead to injury because it is an essential factor in integrated movement, joint coordination, and muscle stiffness [4].

Proprioception is a part of the somatosensory system responsible for sending information to the CNS to monitor various body parts relative to each other [4]. Proprioception is also responsible for receiving signals from afferent neurons, controlling neuromuscular signals, and processing information from stimuli to the CNS, which (by using afferent information provided by proprioceptors) receives and passes on efferent messages to muscles to generate muscle contraction or relaxation [5].

Proprioception comprised two elements: joint position sense and kinesthesia (the sense of limb movement) [5]. Joint position sense is one the components of proprioception and defined as the ability of the subject to perceive a presented joint angle position and (when the limb has been moved) to actively or passively reproduce the joint angle [6]. Muscle receptors may play a vital role in the mid-range of motion, whereas ligament receptors are more sensitive near the end limits of a joint's motion [7].
Knee joint proprioception is mediated primarily through both joint and muscle receptors [8]. Studies have demonstrated the effect of afferent information from the knee joint in the neuromuscular control of the limb [7, 8]. According to Johansson et al., the joint receptors through the gamma motor system provide information that helps to modify muscle tone around the knee [8]. The knee is the largest joint in the human body, and it is also the most complicated one [4]. The quadriceps muscles are vital in keeping the knee joint stable and dynamic [4]. Knee joint proprioception seems to be of particular importance, as it provides the most stable postural position [4].

According to some studies, body sway increases when proprioception is disrupted during standing [9]. On the other hand, deficits in proprioception primarily cause balance impairments in people with mild MS. Indeed ability to maintain static postural position decreases in people with MS [9]. Proprioception requires instant and coordinated communication between CNS and peripheral nervous system. While in MS patients, connections between the brain and other parts of the body are disrupted [10]. Thus these people are susceptible to injury. We aimed to find out the differences between MS patients and healthy control groups regarding their position sense of knee joint.

\section{Materials and Methods}

\section{Study Subjects}

Ten patients with MS (both genders) referred to Sina Hospital, and 10 healthy subjects (bot genders) participated in this case-control study. The patients were examined for disability by Expanded Disability Status

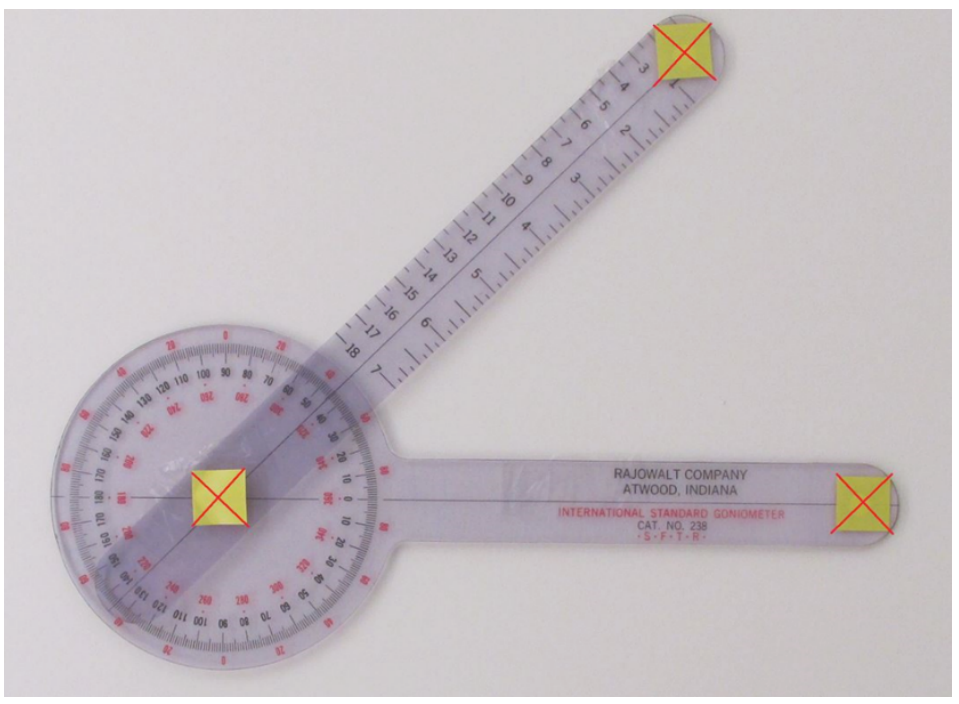

Figure 1. A photo from a goniometer in $45^{\circ}$ at first trial 
Scale (EDSS) and depression by Patient Health Questionnaire-9 (PHQ-9). The inclusion criteria were the following: age between 20-35 years, $\mathrm{PHQ} \leq 15$ indicating the subjects were not in a severe depression, and EDSS $\leq 5$ indicating the disability of the subjects was severe enough to impair their full daily and work activities, for instance, unable to walk without aid or rest for $200 \mathrm{~m}$. Also, subjects had no history of anemia, diabetes, hypothyroidism, surgery, fracture, cardiopulmonary and neuromuscular diseases. The subjects would be excluded from either group if they experienced any fatigue or stress before testing. All subjects signed an informed consent approved by the institutional Ethics Committee of Tehran University of Medical Sciences.

\section{Experimental protocol}

For assessing the validity and reliability of the camera (canon 16 megapixel, Japan), a goniometer was put on the front wall, and photos were captured from 15, 30, $45,60,75$ degrees of a goniometer in four consecutive trials. Then the errors of the camera were calculated by determining the location of the reflective marker, which was attached to the goniometer, according to Figure 1.

In our study, we used the goniometric evaluation accompanied by camera photography for assessing proprioceptive acuity of the knee joint because this method is an accurate method for measuring the joint angle [10]. Four reflective markers attached to the greater trochanter of femur, lateral condyle of femur, head of the fibula, and lateral malleolus of the dominant limb were defined as the limb used to kick a ball (Figure 2). Then, the subject with the eyes closed sat on a chair while his or her dominant knee was at 90 flexion, and a stool supported the non-dominant limb. One camera (Canon camera 16-megapixel) was placed at a distance of $80 \mathrm{~cm}$ and height of $70 \mathrm{~cm}$ from the subject (Figure 1).

The therapist performed passive knee extension of the dominant limb from a starting position (knee $90^{\circ}$ flexion) to a target angle $\left(45^{\circ}\right)$ determined using a goniometer. After holding the target position for 5 seconds, the subject was asked to return his or her limb to the starting position. Then, the subject was asked to reproduce the desired angle actively, and whenever he or she felt that the knee reached from 90 to 45 degrees, he or she pointed a laser on the front wall. Because the subject's eyes are closed and speech may affect the case's position sense, whenever the subject repositions the angle of knee joint, he or shows this repositioning to the therapist by a laser, not by word or movement. So, when the subject points a laser, we know that it is the moment for

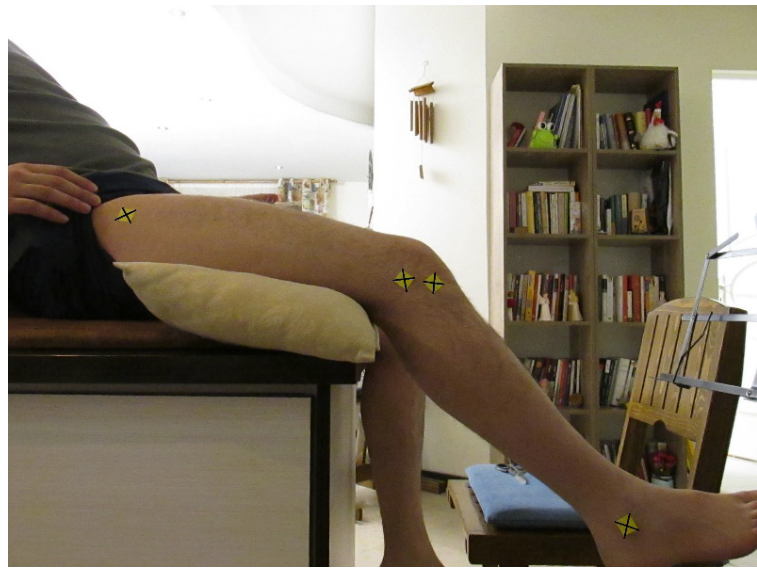

Figure 2. Reflective marks

$J \mathrm{MR}$

Landmarks: greater trochanter of femur, lateral condyle of femur, head of the fibula, and lateral malleolus of the dominant limb

capturing the photo. At that moment, a photo is immediately captured by a camera. In the next trials, the subject actively reproduces knee angle in four consecutive trials (Figure 3). In this study, the reproduction of angle is performed actively because active reproduction is more accurate than passive reproduction. In active testing, the input from muscle receptors is maximized, and active testing is more functional than passive testing [6]. In the first trial, reproducing angle was done by the therapist passively for cases to show them how to do it. In the next tests, the patients were asked to reposition the angle actively.

\section{Study analysis}

To determine the position of reflective markers in the space, we measured three angles: between the femur and horizontal line, middle $90^{\circ}$, and between shin and perpendicular line. The first angle was calculated with the ratio of opposite side to adjacent side (yellow side to adjacent red). The second angle is $90^{\circ}$ and is constant. The third angle was calculated with the ratio of adjacent side to opposite side (red to yellow). The sum of these three angles was the angle of knee joint. The ratio of two sides should be calculated from the arctan method in radians, and then converted to degrees. They were obtained in the software by determining the location of the $\mathrm{X}$ and Y markers.

Absolute, variable, and constant repositioning errors were explained in detail as follows:

1. The absolute angular error is defined as the absolute difference between the target position and the esti- 


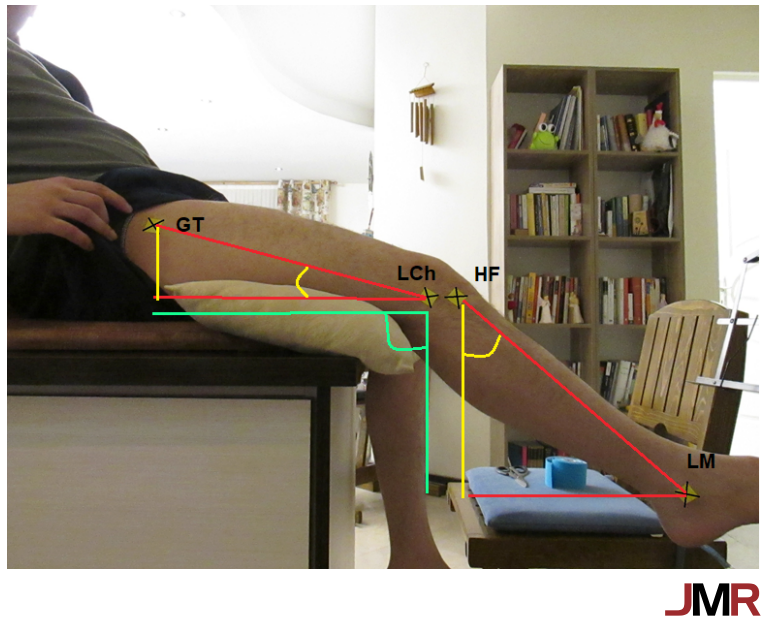

Figure 3. Calculating the angle of the knee

GT, greater trochanter; $\mathrm{LCh}$, lateral condyle of femur; HF, head of the fibula; LM, lateral malleolus

mated position. It measures the difference between the measured angle and the target angle without taking into consideration the direction of error (Equation 1).

2. The constant angular error measures the difference between the measured angle and the target angle, taking into consideration the direction of error (Equation 2).

3. The variable angular error is represented by the standard deviation from the mean of a set of response errors and was determined as the standard deviation from the mean of the constant errors (Equation 3) [4]. According to the following formulas, variables of this study were evaluated:

Equation 1: Absolute error $=\sum^{\left|X_{-} C\right|}{ }_{K}$

Equation 2: Constant error $=\sum^{(x-C) / K}$

Equation 3: $\sqrt{\left(\sum^{(X-C)^{2}} / /\right)-C E^{2}}$
In these three equations, $\mathrm{X}$ indicates the target angle, $\mathrm{C}$ refers to the measured angle, $\mathrm{K}$ is the number of repetitions, and $\mathrm{CE}$ is the constant error.

The obtained data were analyzed in SPSS V. 22. Mean and standard deviations were calculated for the variables under study. The Independent $t$ test was used to compare constant, absolute, and variable repositioning errors between the two groups. The significance level of 0.05 was used for the statistical analyses.

\section{Results}

The Table 1 presents the validity and reliability of the camera in 5 angles and four consecutive trials. Table 2 presents the demographic characteristics of the participants. It shows no significant differences between the two groups in height, weight, and depression rate. However, two groups were significantly different regarding their mean age $(\mathrm{P}=0.03)$. Since there is no correlation between age and repositioning errors, it is not a covariant factor. Table 3 presents the mean, standard deviation, $\mathrm{P}$-value, effect size, and confidence interval values for repositioning error.

$$
\text { Equation 4: effect size }=\frac{\text { Mean }_{\text {control }}-\text { Mean }_{M S}}{\text { Total standard deviation }}
$$

According to Table 3, the absolute, variable, and constant errors of repositioning angle, which represent the accuracy of repositioning, were not significantly different between the two study groups.

\section{Discussion}

This study aimed to compare the position sense of knee joint in people with MS and healthy subjects. The deficits in proprioception cause mobility and balance impairment, falls, injury, and reduction in the quality of life. Based on our results, there were no significant differ-

Table 1. Validity and reliability of the camera

\begin{tabular}{cccccccccc}
\hline Angles & Trial 1 & Trial 2 & Trial 3 & Trial 4 & Mean & Error & Absolute Errors & Correlation \\
\hline 15 & 17.53 & 17.32 & 17.55 & 17.43 & 17.46 & -2.46 & 2.46 & 0.99 \\
\hline 30 & 32.86 & 32.74 & 32.88 & 32.93 & 32.85 & -2.85 & 2.85 & 0.99 \\
\hline 45 & 46.73 & 46.55 & 46.56 & 46.47 & 46.58 & -1.58 & 1.58 & 0.99 \\
60 & 61.71 & 61.63 & 61.9 & 61.77 & 61.75 & -1.75 & 1.75 & 0.99 \\
75 & 77.05 & 77.01 & 76.9 & 76.96 & 76.98 & -1.98 & 1.98 & 0.99 \\
\hline
\end{tabular}


Table 2. Demographic characteristics of the participants

\begin{tabular}{|c|c|c|c|c|}
\hline Parameter & Group & Mean \pm SD & Range & $\mathbf{P}$ \\
\hline \multirow{3}{*}{ Age (y) } & Control & $27.60 \pm 3.71$ & $20-33$ & \multirow{3}{*}{0.03} \\
\hline & & & & \\
\hline & MS & $31.40 \pm 3.50$ & $25-35$ & \\
\hline \multirow{2}{*}{$\begin{array}{l}\text { Height } \\
(\mathrm{cm})\end{array}$} & Control & $167.70 \pm 8.21$ & $157-181$ & \multirow{2}{*}{0.19} \\
\hline & MS & $163 \pm 7.21$ & $151-179$ & \\
\hline \multirow{3}{*}{$\begin{array}{l}\text { Weight } \\
\text { (kg) }\end{array}$} & Control & $63.80 \pm 10.26$ & $54-82$ & \multirow{3}{*}{1} \\
\hline & & & & \\
\hline & MS & $63.80 \pm 17.29$ & $44-93$ & \\
\hline \multirow{2}{*}{$\begin{array}{c}\text { Depression } \\
(0-27)\end{array}$} & Control & $3.40 \pm 1.77$ & $1-6$ & \multirow[b]{2}{*}{0.05} \\
\hline & MS & $740+350$ & $2-12$ & \\
\hline
\end{tabular}

ences in position sense between MS people and healthy subjects.

The findings of this study are in agreement with the results of Fling BW et al. (2015). Their results showed similar postural adaptation between patients with MS and age-matched control participants, despite overall deficits in postural motor control of patients with MS. Moreover, patients with MS demonstrated better retention the following day [11]. However, the results of this clinical study are inconsistent with many published studies. Recently, BW Fling et al. reported reduced white matter microstructural integrity of the cortical proprioceptive tracts and poorer balance control on proprioceptive-based tasks in patients with MS compared with agematched healthy controls [9].

The results of this study disagree with the results of Rougier et al. study that found a statistically significant differences in the center of gravity and its vertical devia- tion from the center of pressure component in patients with MS. Rougier et al. expressed the necessity for the ataxo-spastic MS group to develop augmented neuromuscular control to justify the body movements, as compared to the spasticity MS group and age-matched healthy adults. This study revealed that ataxo-spastic MS patients, affected by proprioceptive loss, could compensate for this deficit with more efficient control strategies when standing still with open eyes [12].

Former studies reveal that balance impairments in people with MS are primarily the result of deficits in "proprioception". Because MS affects the ability of nerves to conduct information, problems with transmitting proprioceptive information from the lower limb joints to the brain (and back again) may play a key role in poor balance control [2].

Table 3. Mean, standard deviation, $P$-value, effect size, and confidence interval values for proprioception variables

\begin{tabular}{|c|c|c|c|c|c|c|c|}
\hline \multirow{2}{*}{ Variable } & \multirow{2}{*}{ Group } & \multirow{2}{*}{ No. } & \multirow{2}{*}{ Mean \pm SD } & \multirow{2}{*}{$\mathbf{P}$} & \multicolumn{2}{|c|}{ 95\% Confidence Interval } & \multirow{2}{*}{$\begin{array}{c}\text { Effect } \\
\text { size }\end{array}$} \\
\hline & & & & & Lower & Upper & \\
\hline \multirow{2}{*}{$\begin{array}{l}\text { Constant } \\
\text { error }\end{array}$} & Control & 10 & $1.55 \pm 0.70$ & \multirow{2}{*}{0.54} & \multirow{2}{*}{-0.64} & \multirow{2}{*}{1.17} & \multirow{2}{*}{0.2842} \\
\hline & MS & 10 & $1.28 \pm 1.17$ & & & & \\
\hline \multirow{2}{*}{$\begin{array}{l}\text { Variable } \\
\text { error }\end{array}$} & Control & 10 & $2.69 \pm 1.21$ & \multirow{2}{*}{0.53} & \multirow{2}{*}{-1.10} & \multirow{2}{*}{2.04} & \multirow{2}{*}{0.2848} \\
\hline & MS & 10 & $2.22 \pm 2.03$ & & & & \\
\hline \multirow{2}{*}{$\begin{array}{l}\text { Absolute } \\
\text { error }\end{array}$} & Control & 10 & $1.55 \pm 0.70$ & \multirow{2}{*}{0.54} & \multirow{2}{*}{-0.64} & \multirow{2}{*}{1.17} & \multirow{2}{*}{0.2842} \\
\hline & MS & 10 & $1.28 \pm 1.17$ & & & & \\
\hline
\end{tabular}




\section{Conclusion}

While eyes were closed in evaluating position sense and afferents were omitted. However, results in comparison of position sense between patients with MS and control group have shown that there are no significant differences in position sense of knee joint in both groups. Impairment of position sense is not a consequence of MS disease, and many factors may affect repositioning errors in the knee joint.

This study has several limitations. First, the physical power of the control group was not controlled, and we assumed that they had similar body strength. But the power of every person is different, which affects the results. Second, repositioning errors of the nondominant leg were not compared. Further studies are required to resolve the limitations of the current study. We suggest that future studies be conducted using an isokinetic device for controlling speed in evaluating the position sense. Also, these studies may use a digital goniometer to increase the accuracy of research. Finally, they can put inclusion criteria for assessing the physical activity in both groups and determine some cut off points for recruiting study subjects.

\section{Ethical Considerations}

\section{Compliance with ethical guidelines}

All ethical principles were considered in this article. The participants were informed about the purpose of the research and its implementation stages; they were also assured about the confidentiality of their information; Moreover, They were allowed to leave the study whenever they wish, and if desired, the results of the research would be available to them.

\section{Funding}

This project was supported by the Rehabilitation School of Tehran University of Medical Sciences.

\section{Authors contributions}

All authors contributed in preparing this article.

\section{Conflict of interest}

The authors declared no conflict of interest.

\section{Acknowledgments}

The authors express their gratitude to the officials and volunteers who contributed to the implementation of this research.

\section{References}

[1] Heine M, van de Port I, Rietberg MB, van Wegen EE, Kwakkel G. Exercise therapy for fatigue in multiple sclerosis. Cochrane Database of Systematic Reviews. 2015; 2015(9):CD009956. [DOI:10.1002/14651858.CD009956.pub2] [PMID]

[2] National Psoriasis. Board of directors meeting scottsdale. [Internet]. 2018 [Update 2018 November 10]. Availble from: https://www.nationalmssociety.org/ About-the-Society/ MS-Prevalence.10/9/2018

[3] Guner S, Inanici F. Yoga therapy and ambulatory multiple sclerosis assessment of gait analysis parameters, fatigue and balance. Journal of Bodywork and Movement Therapies. 2015; 19(1):72-81. [DOI:10.1016/j.jbmt.2014.04.004] [PMID]

[4] Mohammadi Bazneshin M, Amiri A, Jamshidi AA, VasaghiGharamaleki B. Quadriceps muscle fatigue and knee joint position sense in healthy men. Specific Physical Therapy Journal. 2015; 5(2):109-14. [DOI:10.15412/J.PTJ.07050207]

[5] Ribeiro F, Mota J, Oliveira J. Effect of exercise-induced fatigue on position sense of the knee in the elderly. European Journal of Applied Physiology. 2007; 99(4):379-85. [DOI:10.1007/s00421-006-0357-8] [PMID]

[6] Ribeiro F, Santos F, Gonçalves P, Oliveira J. Effects of volleyball match-induced fatigue on knee joint position sense. European Journal of Sport Science. 2008; 8(6):397-402. [DOI:10.1080/02614360802373060]

[7] Gear WS. Effect of different levels of localized muscle fatigue on knee position sense. Journal of Sports Science \& Medicine. 2011; 10(4):725. [PMID] [PMCID]

[8] Hiemstra LA, Lo IK, Fowler PJ. Effect of fatigue on knee proprioception: Implications for dynamic stabilization. The Journal of Orthopaedic and Sports Physical Therapy. 2001; 31(10):598-605. [DOI:10.2519/Journal of Orthopaedic \& Sports Physical Therapy.2001.31.10.598] [PMID]

[9] Fling BW, Dutta GG, Schlueter H, Cameron MH, Horak FB. Associations between proprioceptive neural pathway structural connectivity and balance in people with multiple sclerosis. Frontiers in Human Neuroscience. 2014; 8:814. [DOI:10.3389/fnhum.2014.00814] [PMID] [PMCID]

[10] Changela PK, Selvamani K. A study to evaluate the effect of fatigue on knee joint proprioception and balance in healthy individuals. Indian Journal of Physiotherapy and Occupational Therapy. 2013; 7(1):213.

[11] Fling BW, Dutta GG, Horak FB. Functional connectivity underlying postural motor adaptation in people with multiple sclerosis. NeuroImage Clinical. 2015; 8:281-9. [DOI:10.1016/j. nicl.2015.04.023] [PMID] [PMCID]

[12] Rougier P, Faucher M, Cantalloube S, Lamotte D, Vinti M, Thoumie P. How proprioceptive impairments affect quiet standing in patients with multiple sclerosis. Somatosensory \& Motor Research. 2007; 24(1-2):41-51. [DOI:10.1080/08990220701318148] [PMID] 